


\section{Docencia colaborativa universitaria: planificar, gestionar y evaluar con entornos virtuales de aprendizaje}





\title{
Docencia colaborativa universitaria: planificar, gestionar y evaluar con entornos virtuales de aprendizaje
}

\author{
Beatriz Rodríguez Martín \\ Coordinadora
}

Ediciones de la Universidad

de Castilla-La Mancha

Cuenca, 2020 
(C) de los textos: sus autores.

(C) de la edición: Universidad de Castilla-La Mancha.

Edita: Ediciones de la Universidad de Castilla-La Mancha

Colección ATENEA no 22

Imagen de cubierta: Photo by Pixabay from Pexels (CC0)

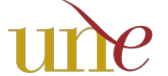

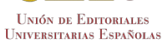

Esta editorial es miembro de la UNE, lo que garantiza la difusión y comercialización de sus publicaciones a nivel nacional e internacional.

ISBN: 978-84-9044-399-6

D.O.I.: http://doi.org/10.18239/atenea_2020.22.00

Composición: Compobell

Hecho en España (U.E.) - Made in Spain (U.E.)

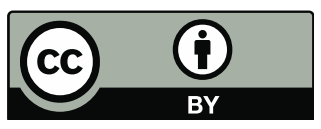

Esta obra se encuentra bajo una licencia internacional Creative Commons CC BY 4.0.

Cualquier forma de reproducción, distribución, comunicación pública o transformación de esta obra no incluida en la licencia Cretative Commons CC BY 4.0 solo puede ser realizada con la autorización expresa de los titulares, salvo excepción prevista por la ley. Puede Vd. acceder al texto completo de la licencia en este enlace: https://creativecommons.org/licenses/ by/4.0/deed.es 


\section{AGRADECIMIENTOS}

Este libro ha sido realizado en marco de un Proyecto de Innovación Docente de la Conferencia Nacional de Decanos de Enfermería. 



\section{Preámbulo}

Nuestra sociedad avanza a pasos de gigante, cambiando el modo que tenemos de relacionarnos con otras personas y de realizar nuestras actividades de la vida cotidiana. En estos cambios es innegable la enorme influencia que están ejerciendo las tecnologías de la información y la comunicación que han cambiado nuestro modo de relacionarnos, comprar, viajar, realizar actividades cotidianas y, como no, aprender.

El mundo académico no es ajeno a estos cambios y la docencia universitaria ha dejado atrás el paradigma en el que el docente era el transmisor de conocimientos primando un nuevo modelo en el que el profesor es el facilitador del conocimiento, mientras que el alumnado y su propio proceso de aprendizaje adquieren un rol protagonista.

Para hacer posible este cambio, uno de los instrumentos clave son los entornos virtuales de aprendizaje, entendidos como espacios docentes alojados en la web que incluyen una serie de herramientas informáticas que hacen posible la interacción durante el proceso de aprendizaje.

Diversos estudios muestran la rapidez con la que el alumnado se adapta a la incorporación de entornos virtuales de aprendizaje a la docencia. En cambio, a pesar de que los docentes universitarios estamos acostumbrados a recibir formación continuada de manera online o semipresencial, ciertos docentes siguen mostrando reticencia a incorporar a su docencia entornos virtuales de aprendizaje, o lo hacen de manera puntual, expresando una cierta disconformidad a salir de la zona de confort que nos proporciona la docencia tradicional. Por poner solo un ejemplo, el alumnado universitario ha respondido muy bien al cambio brusco, debido a la crisis del coronavirus, de 
la docencia presencial a la docencia virtual. A los docentes nos ha costado un poco más adaptarnos a los entornos virtuales, especialmente cuando no había habido experiencia o formación previa. Aunque como dice el refranero español, la necesidad agudiza el ingenio y la necesidad hace maestros. Y esta crisis ha servido para que todos los docentes se vean obligados a experimentar la docencia en entornos virtuales. En este proceso han ayudado mucho las experiencias previas realizadas en el ámbito universitario (muchas universidades contaban ya con sistemas de videoconferencia, plataformas como Microsoft Teams, Campus Virtual...) y el trabajo a contrarreloj de los servicios de informática que han hecho más sencillo el reto al que nos enfrentamos de pasar en unos días de la docencia presencial a la docencia online.

A pesar de que es bien conocida la utilidad y las ventajas de los entornos virtuales de aprendizaje, en especial para el aprendizaje colaborativo, ciertos docentes continúan utilizando alguno de estos entornos como un mero repositorio de conocimientos o no aprovechan todo su potencial.

Aunque no existen recetas mágicas que nos aseguren el éxito cuando utilizamos entornos virtuales de aprendizaje para el aprendizaje colaborativo, el objetivo de este libro es mostrar experiencias de buenas prácticas que ayuden al lector en el empleo de los entornos virtuales de aprendizaje, independientemente de la disciplina en la que se que se quieran aplicar.

Este libro no pretende ser un manual de uso de ciertas herramientas en concreto, para eso tenemos múltiples tutoriales en redes sociales, sino analizar experiencias prácticas de aplicación de entornos virtuales de aprendizaje y aprendizaje colaborativo en la docencia universitaria que ayuden a otros docentes a planificar, gestionar y evaluar las actividades docentes en estos entornos con cierto éxito.

Esperamos que alguna de las experiencias que aparecen en este libro sirva de inspiración a los docentes para mejorar nuestras competencias relacionadas con la docencia en entornos virtuales de aprendizaje. 


\section{Índice}

CAPítulo i. Entornos virtuales de aprendizaje y APRENDiZAJE COLABORATIVO EN UNA SOCIEDAD QUE AVANZA ...................... Beatriz Rodriguez Martín

Capítulo 2. ¿Cómo motivar a los alumnos en el trabajo COLABORATIVO? RESOLUCIÓN DE CONFLICTOS

María del Carmen Zabala Baños

Capítulo 3. Evaluación de las actividades en los entornos VIRTUALES DE APRENDIZAJE

Esmeralda Santacruz Salas

Capítulo 4. Claves del ÉXito para la Planificación de actiVIDADES EN REDES SOCIALES

Carlos Alberto Castillo Sarmiento

Capítulo 5. Desafíos al docente universitario: las redes SOCIALES EN EL MUNDO ACADÉMICO

José Luis Martín Conty

Capítulo 6. La gamificación y los Sistemas de Respuesta INTERACTIVA COMO HERRAMIENTAS PARA REFORZAR EL APRENDIZAJE COLABORATIVO Y COOPERATIVO EN EL AULA .....................

Rubén Mirón González 
Capítulo 7. Experiencia de aplicación del aprendizaje COLABORATIVO CON ESTUDIANTES DE ENFERMERÍA FAMILIAR Y

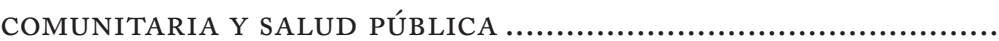
Montserrat Pulido Fuentes

Capítulo 8. Buenas prácticas en entornos virtuales de APRENDIZAJE BASADOS EN WIKIS

Beatriz Rodríguez Martín

Capítulo 9. Buenas prácticas en entornos virtuales basaDOS EN BLOGS

Carlos Alberto Castillo Sarmiento 


\section{Recursos}

\section{ÍNDICE DE TABLAS}

Tabla 1. Matriz de una rúbrica de evaluación................................ 44

Tabla 2. Rúbrica ejemplo 1: Actividad Diagnóstica ...................... 46

Tabla 3. Rúbrica ejemplo 2: Actividad Formativa ........................ 47

Tabla 4. Rúbrica ejemplo 3: Actividad Sumativa ......................... 48

Tabla 5. Temario de la asignatura Enfermería Médico-Quirúrgica 179

Tabla 6. Evaluación de seminarios/laboratorios de Enfermería Médico-Quirúrgica 1 del curso 2017-2018.....................

\section{ÍNDICE DE FIGURAS}

Figura 1. Modelo conceptual sobre los diferentes tipos de evaluación 38

Figura 2. Modelo Kirkpatrick......................................................... $\quad 39$

Figura 3. Esquema de herramientas de evaluación en entornos virtuales de aprendizaje...................................................... 42

Figura 4. Secuenciación de dinámicas por bloque temático............ $\quad 80$ 



\title{
Capítulo 4 Claves del éxito para la planificación de actividades en redes sociales
}

\author{
Carlos Alberto Castillo Sarmiento \\ Universidad de Castilla-La Mancha \\ http://doi.org/10.18239/atenea_2020.22.04
}

\section{REDES SOCIALES Y DOCENCIA UNIVERSITARIA}

En la actualidad nos encontramos con un conjunto de alumnos que están muy acostumbrados a emplear las redes sociales. Bueno, más bien podríamos decir que prácticamente viven en las redes sociales: se informan a través de ellas, se comunican a través de ellas, contactan con sus conocidos a través de ellas... entonces, ¿`i usan tanto las redes sociales no sería conveniente incorporarlas de alguna forma a la docencia? Sobre este tema, hay un cierto debate en los entornos docentes. Por un lado, los docentes que tenemos una cierta experiencia en docencia también tenemos una cierta edad y por tanto estamos mucho menos acostumbrados que nuestros alumnos las redes sociales y, evidentemente, a nadie le gusta salir de su zona de confort. Por otro lado, surge la duda de si resulta conveniente o no cambiar el medio en el que se disemina la información solo porque sea un medio muy empleado por los alumnos.

En mi opinión, este debate está zanjado ya que, por un lado, no debería ser un reto para un buen docente ser capaz de adaptarse a un nuevo medio de comunicación, pero, además, poner de excusa que es el medio favorito de los alumnos para comunicarse para no emplear estas herramientas se parece demasiado al "esto siempre se ha hecho así" que tan pocas alegrías nos ha dado. Además, en mi caso y en el de muchos otros compañeros, me dedico a la docencia universitaria en una universidad pública por lo que producir, al menos, parte del contenido de una asignatura en un formato abierto es una cuestión interesante tanto porque personas ajenas a la docencia universitaria pueden aprovechar este conocimiento, cómo por la transparencia que el ejercicio de la docencia que implica. 
Este último punto abre una reflexión interesante acerca de hasta dónde debe llegar el compromiso con nuestros alumnos. En muchos campos actualmente el conocimiento se recicla a una velocidad de vértigo por lo que, entra dentro de lo posible, que los contenidos que un alumno adquirió en el primer curso de su vida universitaria hayan quedado, al menos, parcialmente obsoletos cuando éste alumno termine su formación universitaria. Por tanto, las actividades realizadas en redes sociales nos permiten mantener el contacto con esos alumnos que no están ahora presencialmente en nuestras clases a la vez que les permitimos que reciclen parte del contenido que antaño desarrollaron.

Además de estas breves reflexiones, hay otros motivos por los que realizar actividades en redes sociales debería ser interesante para los docentes universitarios. Por un lado, este tipo de actividades en abierto aumentan el valor de nuestro trabajo, es decir, es una forma de resaltar la importancia de las cosas que hacemos en la universidad. Pero, por otro lado, introduce una variable interesante que quizá nunca nos habíamos planteado. Imaginemos por ejemplo que somos profesores de la asignatura de Farmacología, nuestros alumnos, en un futuro, sean de la rama de Ciencias de la Salud que sean, tendrán que defender de alguna forma el uso de fármacos específicos contra una determinada patología. Evidentemente todos los fármacos tienen efectos secundarios más o menos graves. Frente al uso de fármacos estos alumnos se encontrarán con gente que defiende el discurso de que las farmacéuticas están para ganar dinero, que no quieren realmente curar enfermedades, etcétera. En el campo académico seguramente este es un debate que está completamente saldado, pero sin embargo es un debate al que estos hipotéticos alumnos tendrán que enfrentarse, ¿no sería por tanto interesante plantear actividades en redes sociales que nos den la posibilidad de contrastar nuestro discurso contra estas actitudes? ¿no resultaría beneficioso para nuestros alumnos comprobar cómo se puede argumentar lo que ellos saben en un entorno menos académico? $\mathrm{O}$, dicho de otra forma ¿tendríamos la posibilidad en una de nuestras clases de tener un debate de este tipo? Por tanto, el desarrollo de actividades de índole variada en redes sociales nos permite interaccionar con personas "reales" de forma mucho más espontánea de lo que pudiera ocurrir dentro de una clase.

\section{COMUNICACIÓN EN REDES SOCIALES}

Decir que Internet ha cambiado la forma de comunicarnos es quedarse un poco corto. Aunque en sus principios Internet no dejaba de ser un sitio donde había mucha información, en la actualidad esa no es solo una de sus principales características. Ahora, además, Internet nos permite comunicar- 
nos entre nosotros, pero este "nosotros" no se refiere solo a personas que se conocen, sino que, normalmente, se refiere a completos desconocidos. Es decir, la Internet actual nos permite formarnos, comunicarnos, colaborar, compartir información y opiniones con personas que son desconocidas, que no tienen por qué vivir cerca de nosotros. De hecho, actualmente cualquier persona es capaz de crear y distribuir contenidos a través de Internet de forma prácticamente gratuita. Esto es lo que se conoce como una comunidad virtual y a esta Internet, la que nos permite crear contenidos, difundirlos y comunicarnos con otros usuarios, es a la que conocemos como Web 2.0.

Dentro de esta Web 2.o unos actores importantísimos son las redes sociales. Las redes sociales han cambiado de hecho la forma de comunicarnos en muchísimos aspectos. Por poner un ejemplo cotidiano no es extraño que en un telediario se comente la opinión de un político que ha expresado a través de un tuit. No obstante, no todas las redes sociales son igual de relevantes y del mismo modo tampoco lo serán en su posible aplicación en el entorno docente. En esta sección describiremos las tres redes sociales más relevantes pensando en su posible aplicación en docencia. Dejaremos de lado una red social importantísima basada en vídeo cómo es YouTube debido a que no es un medio muy adecuado para que la información fluya en ambos sentidos, sino más bien solo desde el vídeo hacia el espectador (aunque es cierto que tiene una herramienta de comentarios).

Por tanto, nos centraremos en Twitter, Facebook e Instagram, redes sociales que además disponen de aplicación móvil que facilita el acceso desde nuestro smartphone. Debemos conocer las posibilidades que nos proporcionan estos entornos, es decir, sus características principales y cómo funcionan, ya que en función de estas podemos plantear un tipo de actividades u otro. Empecemos.

\section{Facebook}

En la actualidad Facebook está viendo descender su número de usuarios $y$, de hecho, no es la preferida de los universitarios, aunque sigue siendo una red social muy potente. Está red social nos permite hacer publicaciones que contengan imágenes, vídeos e hipervínculos a otras webs, así como una cantidad de texto suficiente para hacer llegar nuestro mensaje con claridad.

En Facebook las relaciones entre usuarios se marcan con una amistad: un usuario le envía a otro una solicitud de amistad y éste puede aceptarla o no. Si la solicitud se acepta, ambos usuarios quedan vinculados y ambos podrán leer las publicaciones del otro, pero si no se acepta, las publicaciones de un usuario 
quedarán ocultas para el otro y viceversa. Esta relación de amistad se concreta en el "timeline" de un usuario, en el que irán apareciendo las publicaciones del otro en orden cronológico inverso y viceversa

No obstante, Facebook nos permite definir varios niveles de privacidad en nuestras publicaciones, siendo el más abierto "público", esto es, que nuestras publicaciones son accesibles para cualquier usuario no solo de Facebook sino de Internet.

Además de la funcionalidad básica de Facebook, que debemos conocer para poder emplear esta herramienta en docencia, hay dos funciones de Facebook que nos podrían interesar y que son mucho menos empleadas. Una de ellas son los grupos, es decir, la posibilidad de generar un espacio privado dentro de Facebook para un número de usuarios seleccionados. Otra opción que puede ser muy interesante en docencia es la posibilidad de, en vez de trabajar con un perfil de Facebook, trabajar con una página de Facebook. Las páginas se diferencian de los perfiles en que no es necesario establecer una relación de amistad para acceder al contenido. Los usuarios de Facebook indican que les "gusta" una página y desde ese momento las publicaciones de esa página aparecen en su "timeline". Es decir, es una forma de crear dentro de Facebook una comunidad de usuarios en torno a unos intereses comunes que se expresan en esa página que siguen.

\section{Twitter}

Twitter es la red de microblogging más empleada y probablemente la red que más experiencias tiene recogidas en la literatura. La principal característica de Twitter es que cada publicación o tuit solo puede contener 280 caracteres como máximo. Esta característica hace que sea una red muy interesante si nosotros por ejemplo nos estamos planteando que nuestros alumnos deben aprender a condensar el mensaje que quieren transmitir. No obstante, cada publicación puede ir acompañada de contenido externo, que puede ser imágenes, animaciones, vídeos... Además, varias publicaciones pueden encadenarse formando lo que se conoce como un "hilo".

Además de la publicación de contenido propio, esta red nos permite también compartir contenido de otros usuarios, lo que se conoce como un retuit, así como incorporar a nuestras publicaciones contenido que proviene de otras redes.

La forma más habitual de comunicarse entre usuarios en Twitter es de forma pública, es decir, cualquier persona podría leer el contenido que estamos publicando o la conversación que estamos manteniendo con otro usuario 
de Twitter y, por supuesto, podría participar en esta conversación o podría realizarnos alguna pregunta o corrección sobre el contenido que estamos publicando. Aunque es mucho menos común, y seguramente también sea menos interesante desde el punto de vista docente, Twitter también nos permite mantener conversaciones de forma privada. Para ello, previamente hemos debido de establecer un vínculo de confianza con otros usuarios y solo serán éstos los que puedan leer y comentar nuestros mensajes. Por último, Twitter nos permite también la posibilidad de comenzar conversaciones con otros usuarios, para ello podemos "mencionar" a un usuario de Twitter en una publicación y éste recibirá un aviso de que se ha recibido esta mención, dándole la oportunidad de intervenir en la conversación.

En Twitter cada usuario dispone de lo que se conoce como un "timeline". Este está configurado por las publicaciones de los usuarios a los que seguimos, es decir, aquellos que hemos marcado en Twitter como interesantes, que se muestran siguiendo un orden cronológico inverso, de las más recientes hacia las más antiguas en el tiempo. Del mismo modo, nosotros también podemos ser seguidos por otros usuarios, de tal forma que nuestras publicaciones se incorporarán a sus respectivos "timelines". Es decir, al contrario que, por ejemplo en Facebook, en Twitter las relaciones entre usuarios suelen ser asimétricas: podemos seguir a un usuario y que éste no nos siga y también nos puede seguir un usuario a quien nosotros no sigamos, todo depende de los intereses de cada usuario.

Por último, Twitter nos proporciona una serie de temas "calientes", los temas de los que más están hablando el resto de los usuarios. Esto es lo que se conoce como trending topic. Pero además podemos buscar aquellos temas que nos interesen, para ello conviene, además de emplear las palabras clave, conocer si la gente que habla de un tema concreto emplea lo que en Twitter se conoce como un "hashtag", es decir, si estos usuarios etiquetan sus publicaciones sobre este tema que nos interesa de una forma determinada.

\section{Instagram}

Es la red social más nueva de las tres y probablemente la preferida por nuestros alumnos. La base de las comunicaciones dentro de Instagram son las fotos, aunque también los vídeos. Inicialmente Instagram se desarrolló para publicar fotos en formato cuadrado, a las cuales se les podría aplicar diversos filtros. No obstante, en cada publicación la foto o el vídeo que se publique admite un texto, aunque, como hemos comentado, todo el diseño de la apli- 
cación gira en torno a la imagen, por tanto, es un aspecto para tener muy en cuenta a la hora de desarrollar actividades en Instagram

Instagram repite la forma interacciones entre los usuarios que ya hemos descrito en redes anteriores. De nuevo podremos trabajar con una cuenta privada o una cuenta pública en función de lo que nos interese, podemos seguir otras cuentas y estás también nos podrán seguir a nosotros configurando así nuestro "timeline”. Igual que ocurría en Facebook, podemos utilizar una cuenta profesional pudiéndose elegir entre un perfil de "creador" o bien "empresa", proporcionandose nuevas funcionalidades para este tipo de cuentas.

\section{SUGERENCIAS PARA INCORPORAR ACTIVIDADES EN REDES SOCIALES A TU DOCENCIA}

En este apartado vamos a proporcionar una serie de consejos generales que debemos tener en cuenta a la hora de plantear actividades docentes en redes sociales. Tengamos en cuenta que este capítulo es de carácter general, por tanto está dirigido a docentes de diversas ramas de conocimiento. Mi consejo es que cuando queráis realizar una actividad específica en redes sociales busquéis qué se ha hecho ya en vuestro campo de conocimiento y qué actividades han funcionado mejor y cuáles han funcionado peor (y por qué). Es una reflexión importante la que hay que hacer al inicio de plantear una actividad en redes sociales, ya que no existe una regla mágica que nos permita saber de antemano si la actividad que vamos a realizar tendrá buena acogida y cumplirá con los objetivos que nos hemos marcado. No obstante, en este capítulo proporcionaremos un buen número de consejos para intentar asegurar que las tasas de éxito sean lo más elevadas posible.

Antes de empezar con cualquier actividad que vayamos a planificar para nuestros alumnos en redes sociales, es conveniente dedicar un tiempo a reflexionar sobre unas cuestiones básicas. La primera que debemos plantearnos es qué tipo de red social vamos a emplear, para ello debemos definir cuál es el objetivo de la actividad que queremos plantear a nuestros alumnos, por ejemplo, si queremos que nuestros alumnos desarrollen un texto complejo seguramente Instagram no sea la red social adecuada. El siguiente paso que debemos plantearnos es qué características va a tener el entorno en el que vamos a trabajar, por ejemplo, ¿hablaremos de una red social con carácter público o privado?, ¿nuestros alumnos van a trabajar de forma individual o por grupos?, ¿qué tipo de actividades concretas van a realizar los alumnos?... Además, debemos plantearnos qué tipo de rol le vamos a dar los alumnos ¿̇olo 
van a leer lo que nosotros subamos?, ¿además podrán publicar su propio contenido?, ¿ivalorarán el trabajo de otros alumnos?, etcétera. Debemos plantearnos también qué contenido vamos a aportar nosotros a esta actividad: ¿ivamos a subir imagen, vídeo, bibliografía?, etcétera. Finalmente, casi lo más importante de todo: tendremos que plantearnos cómo vamos a valorar la actividad.

$Y$ es que las redes sociales nos proporcionan a los docentes la oportunidad de incrementar la interacción con nuestros alumnos y el compromiso de éstos con nuestras asignaturas. Además, las redes sociales nos pueden ayudar porque ya disponen de mucho contenido publicado previamente al que podemos recurrir. Pasemos por tanto a desgranar consejos concretos para realizar con éxito actividades en redes sociales.

\section{Conoce tu entorno de trabajo}

Es fundamental para poder desarrollar actividades con éxito en redes sociales conocer de forma avanzada cómo funcionan estás redes. Lo normal es que nuestros alumnos ya sepan utilizarlas, pero probablemente si les pedimos que hagan algo que se salga un poco de lo común no sabrán desarrollar esta tarea y nos plantearán sus dudas a las cuales, evidentemente, no sabremos responder si no somos usuarios con un cierto bagaje en el manejo de la red social correspondiente.

Por otro lado, conocer las redes que estamos usando en profundidad nos permiten saber con qué tipo de herramientas podemos contar para trabajar y con cuáles no. Por tanto, esto nos permitirá desarrollar actividades de forma más precisa para alcanzar nuestros objetivos docentes.

Además, si no conocemos razonablemente la red en la que queremos trabajar, sencillamente, no seremos capaces de comunicarnos con nuestros alumnos al no conocer la terminología necesaria para comunicarnos en una red social.

\section{Conviértete en usuario}

No solo debemos conocer la red social que queremos emplear en nuestras actividades docentes, sino que además debemos estar acostumbrados a usarla. Este uso cotidiano nos permitirá ir recopilando recursos que están disponibles ya en muchas redes sociales. Pero, además, si estamos interesados en que nuestros alumnos empleen redes sociales desde un punto de vista profesional, ellos deben estar acostumbrados a vernos a nosotros emplearlas también. Vamos, que no nos queda otra que ser proactivos. 


\section{Establece unas reglas}

Las redes sociales proporcionan un ambiente mucho más distendido que una clase en la universidad. No obstante, aunque no nos encontremos en un entorno sujeto a las mismas reglas que tenemos en clase, esto no quiere decir que no se deben respetar una serie de reglas, digamos, de convivencia.

De este modo, debemos crear una serie de reglas y avisos que le recuerden a los usuarios que la actividad que estamos desarrollando conlleva un uso específico de la red social y por tanto los usuarios que interaccionan con nosotros se hacen responsables del contenido de sus publicaciones. Es un punto importante ya que, por ejemplo, un usuario podría cometer un delito al publicar una imagen de un libro de texto en una red social y sin embargo este hecho no tendría ninguna repercusión si esa imagen la insertara en una presentación que realizara en una clase.

En cualquier caso, no se trata solo de establecer unas reglas que nos eviten problemas éticos o legales. Lo que intentamos de escribir en este apartado es que mantener la profesionalidad tanto en un entorno presencial como en uno virtual debe ser igual de importante.

Seguramente este tipo de cláusulas no serían necesarias si todos aceptáramos que nuestros avatares en las redes sociales nos representan, de hecho, cada vez es más común en los procesos de selección de empresas se consulten las redes sociales de los candidatos en busca del tipo de personas que son online.

\section{Usa ejemplos de la red social durante tus clases}

Las actividades en redes sociales además pueden ayudarnos a mejorar la experiencia de los alumnos en nuestras clases presenciales. De tal forma que, si mostramos publicaciones de redes sociales durante nuestras clases presenciales, no solo animaremos a los alumnos a participar en las actividades que estamos planteando en las mismas, sino que también seremos probablemente capaces de abrir un debate sobre la experiencia de los alumnos con el uso de las redes sociales y sus opiniones con respecto a los temas que se plantean.

No obstante, hemos de tener en cuenta que esto también altera las dinámicas normales de las clases presenciales, por lo que debemos valorar cuánto tiempo le debemos dedicar a este tipo de mini actividades.

En este sentido, otra opción a valorar en el uso de las redes sociales es abrir la posibilidad de que en una clase presencial mientras ésta se está presentando un material o bien en la videoconferencia o similar, los alumnos no puedan interrumpir para realizar preguntas, sino que estas preguntas deban 
dirigirse a través de la red social de nuestra elección. De esta forma al final de la presentación o de la charla, podremos recopilar todas las preguntas de los alumnos sin haber interrumpido nuestra presentación y sin haber perdido ninguna pregunta.

\section{Usa las redes sociales como fuente de información}

En la actualidad, gran parte de la población incluidos nuestros alumnos, obtienen mucha información a través de las redes sociales. No obstante, para ellos tampoco es sencillo cribar entre toda la información disponible cuál es la más acertada o verídica. Por tanto, podemos usar nuestras actividades en redes sociales para enseñarles a los alumnos cuáles son las páginas o usuarios de referencia de nuestra materia, así como qué herramientas podemos emplear para cribar la información en redes sociales.

\section{Incorpora a tus redes sociales contenido actual}

No es nada extraño que durante el desarrollo de un curso aparezcan noticias relevantes relacionadas con la materia que estamos impartiendo. Compartir o comentar estas noticias a través de nuestras redes sociales permite a los alumnos contextualizar las cuestiones que estamos desarrollando en el aula, es decir, es la respuesta a la ya típica pregunta “¿y esto para qué sirve?”.

\section{Crea una comunidad}

Si la red social que estás usando lo permite, crea un grupo que contenga a tus alumnos. De esta forma habrá un flujo de comunicación directa del cual todos los estudiantes podrán participar. Además de crear una cierta sensación de comunidad, seguramente sea más sencillo para los alumnos comunicarse por este método, dado que las redes sociales son usadas desde dispositivos móviles, que no consultando el correo electrónico.

\section{Anima a los estudiantes a ser creativos pero concisos}

Seguramente la creatividad a la hora de expresar conocimiento sea una competencia que todos nuestros alumnos deben adquirir independientemente de la rama de conocimiento, además, la expresión de este conocimiento debe realizarse de forma concisa, es decir, debemos conseguir expresar lo que tenemos de forma coherente, pero sin aburrir a nuestro interlocutor. Para ello la red social Twitter es ideal pues presenta espacio limitado en cada publica- 
ción, no obstante, esta misma regla puede extrapolarse a cualquier otra red social que queramos emplear.

\section{Usa las redes sociales para hacer encuestas informales}

Esta experiencia puede realizarse tanto de forma asíncrona como cuando tenemos a los alumnos de forma presencial en nuestra clase, es decir, en vez de pedirles que levanten la mano para ver qué opinan como les podemos pedir que respondan a una pregunta a través de la red social. De esta forma podemos controlar de forma más precisa el tanto por ciento de respuestas de cada una de las opciones y aprovechar para proporcionar feedback sobre cada una de las respuestas.

\section{Las redes sociales pueden ser una buena forma de promover reflexión y pen- samiento crítico}

Bastantes estudios de la literatura afirman qué es posible emplear redes sociales tanto para promover la autorreflexión o la reflexión grupal en torno a una actividad, así como para desarrollar el pensamiento crítico de nuestros alumnos. Es por tanto importante que intentemos establecer la forma, si no lo hacemos en nuestras clases presenciales, de que nuestros alumnos reflexionen en este contexto virtual. De hecho, solo tendríamos que canalizar lo que ya hacen hacia nuestros intereses, ya que muchos de nuestros alumnos utilizan las redes sociales para expresar sus puntos de vista sobre determinados temas de forma habitual.

\section{CONCLUSIÓN}

Las redes sociales son potentísimas herramientas para compartir información y comunicarse entre diversos usuarios, estando además su uso ya muy extendido entre nuestros alumnos y siendo prácticamente gratuito. Existen en la literatura bastantes experiencias acerca del uso de redes sociales en docencia que avalan su empleo para promover el aprendizaje de los estudiantes en contextos más informales, así como para que nuestros propios estudiantes sean capaces de desarrollar sus propios entornos de aprendizaje en base a sus propios intereses. Además, las redes sociales nos permiten la posibilidad de incorporar agentes externos a nuestra docencia, proporcionando un contexto más realista que el puramente académico.

No obstante, debemos tener en cuenta que el uso de redes sociales en docencia también incluye algunas desventajas. Algunas de estas desventajas 
están incluidas en la literatura, cómo pueden ser las propias limitaciones de las redes sociales o las limitaciones éticas ya comentadas en este capítulo, además, una limitación importante que no suele reflejarse en la literatura es que el empleo de estas herramientas supone para el docente una importante carga de trabajo adicional. Debemos por tanto valorar todas estas características a la hora de planificar nuestras actividades docentes en redes sociales.

\section{BIBLIOGRAFÍA}

Baumann, P. (2009, enero 16). I40 Health Care Uses for Twitter. Recuperado 8 de diciembre de 20I9, de Phil Baumann website: https://philbaumann. com/2009/oI/16/140-health-care-uses-for-twitter/

Polinario, J. (2016). Cómo divulgar ciencia a través de las redes sociales. CreateSpace Independent Publishing Platform.

Rodríguez-Martín, B., \& Castillo, C. A. (2017). Hidratos de carbono y práctica deportiva: Una etnografía virtual en Twitter. Nutrición Hospitalaria, 34(I), I44I53. doi: $10.20960 /$ nh.990

Rodríguez-Martín, B., \& Castillo, C.A.(2019). Entornos virtuales de aprendizaje: Posibilidades y retos en el ámbito universitario. Recuperado de http://doi.org/Io.I8239/ atena.I4.2019 
